\title{
qPCR primers
}

\begin{tabular}{|c|c|c|}
\hline Gene & Forward (5' to 3') & Reverse (5' to 3') \\
\hline$E B V \_B Z L F 1$ & AATGCCGGGCCAAGTTTAAGCAAC & TTGGGCACATCTGCTTCAACAGGA \\
\hline$E B V \_B R L F 1$ & TGGCTTGGAAGACTTTCTGAGGCT & AATCTCCACACTCCCGGCTGTAAA \\
\hline$E B V \_B M R F 1$ & ATACGGTCAGTCCATCTCCT & CACTTTCTTGGGGTGCTT \\
\hline$E B V \_B A L F 5$ & GCGGCCCCGGAGTTGTTA & CGTGGCCGTGGATCATTATTTC \\
\hline$E B V \_B N R F 1$ & GCAAACATACAGGAGGAAAG & CAGCAGGTTCTCAGCAATC \\
\hline$E B V \_B L L F 1$ & GCCTTGGAGAATATAACCTTG & CATTACTGTCTCGGGTCTTGG \\
\hline$E B V \_B c L F 1$ & GTGGATCAGGCCGTTATTGA & CCTCAAACCCGTGGATCATA \\
\hline$E B V \_E B N A 1$ & TGAGTCGTCTCCCCTTTGGA & CCTTAGCGGGCCAGGTTGTG \\
\hline KSHV_ORF50 & CCTTCGGCCCGGGGTCT & CGGTGGCAGTTGCGTATACTCT \\
\hline$K S H V \_K 8$ & CCTGGACGCTCTCTCACACA & GGATCTGCGAGTTGGAAGCT \\
\hline KSHV_ORF26 & AGCCGAAAGGATTCCACCAT & TCCGTGTTGTCTACGTCCAG \\
\hline KSHV_LANA & TTACCTCCACCGGCACTCTT & GGATGGGATGGAGGGATTG \\
\hline$E B N A \_1$ & GCCGGTGTGTTCGTATATGG & CAAAACCTCAGCAAATATATGAG \\
\hline$E B N A \_2$ & CCACAATGTCGTCTTACACC & ATAACAGACAATGGACTCCCT \\
\hline PR-ORF50 & ATGAAGATGTGGTAGAGCCA & TAGCGCCATCTCTGCCCCCC \\
\hline PR-ORF59 & САСАСТТССАССТССССТАА & CGCACAGAGAAATCACAGGA \\
\hline OriLyt & CCTACATGGGCAGCTTGTCC & TGCTGCCGGGGCTCCTCGTT \\
\hline PLK1 & CCA ACA CCA CGA ACA CGA & GCC TCT TGT CTC TTA GAT TTG GTC \\
\hline GAPDH & GCC TCT TGT CTC TTA GAT TTG GTC & TAG CAC TCA CCA TGT AGT TGA GGT \\
\hline \multicolumn{3}{|c|}{ shRNA sequence } \\
\hline PLK1 ShRNA & GTT CTT TAC TTC TGG CTA TAT & \\
\hline NT ShRNA & CAC AAA CGC TCT CAT CGA CAA G & \\
\hline
\end{tabular}

